\title{
FACTORES ASOCIADOS A VIOLENCIA FÍSICA RECIENTE DE PAREJA HACIA LA MUJER EN EL PERÚ, 2004-2007
}

\author{
Dora Blitchtein-Winicki ${ }^{1, a}$, Esperanza Reyes-Solari ${ }^{1, b}$
}

\begin{abstract}
RESUMEN
Objetivos. Identificar los factores asociados de violencia física reciente (en el último año) de pareja, hacia la mujer en el Perú. Materiales y métodos. Se realizó un análisis secundario de la encuesta demográfica y de salud familiar (ENDES) 2004-2007. Se incluyó a 12257 mujeres unidas o casadas que respondieron al módulo de violencia, con esta información se realizó la categorización de antecedente de violencia física infringida por la pareja. Se llevaron a cabo análisis descriptivos y regresiones logísticas univariadas y multivariadas para muestras complejas. Resultados. La prevalencia de violencia física a nivel nacional fue de 14,5\%, con variación entre regiones geográficas $(9,7$ a 18,9\%). El factor asociado con un mayor riesgo es que la pareja se embriague con frecuencia (OR: 7,2; IC 95\%: 5,4-9,6) y que la pareja controle o limite las visitas de la mujer a familiares o amigos (OR: 4,1; IC 95\%: 3,3-5,0); otros factores asociados son el antecedente de agresión física del padre hacia la madre, el haber tenido uniones previas de pareja, ser conviviente, tener mayor nivel de educación que la pareja o el desbalance en la toma de decisiones en la pareja. Entre los factores asociados con un menor riesgo, estuvieron la comunicación y respeto dentro de la pareja (OR: 0,3 ; IC 95\%: 0,2-0,3) y el que la mujer tenga algún tipo de seguro de salud. Conclusiones. Diversos factores individuales, de relaciones de pareja u otros factores, están asociadas con una mayor probabilidad de violencia de pareja contra la mujer. Otros factores, como la comunicación y el respeto en la pareja, podrían tener carácter protector.
\end{abstract}

Palabras clave: Violencia doméstica; Diversidad cultural; Ubicaciones geográficas; Encuestas epidemiológicas; Violencia contra la mujer; Perú (fuente: DeCS BIREME).

\section{FACTORS ASSOCIATED TO RECENT INTIMATE PARTNER PHYSICAL VIOLENCE AGAINST WOMEN IN PERU, 2004-2007}

\begin{abstract}
Objectives. To identify factors associated to recent (during the last year) intimate partner physical violence against women in Peru. Materials and methods. Using the demographic and Family Health National Survey (ENDES) 20042007 a secondary analysis was performed. 12,257 women married or living with a couple who answered the violence module were included, with this information they were assigned to a category according to the history of having been physically assaulted by their couples. Descriptive analysis and univariate and multivariate logistical regression for complex samples were done. Results. Prevalence of physical violence at the national level was $14,5 \%$, with variations according to the geographical areas $(9,7 \%$ to $18,9 \%)$. The factor associated to a higher risk is having a couple who frequently gets drunk (OR: 7,$2 ; 95 \% \mathrm{Cl}: 5,4-9,6)$ and having a couple controlling or limiting the woman's visits to family or friends (OR: 4,$1 ; 95 \% \mathrm{Cl}: 3,3-5,0$ ); other associated factors are history of physical aggression of the father to the mother, having had previous partners, be cohabiting, having a higher educational level than the partner and having a disbalanced decision taking in the couple. Among the factors associated with a lower risk are the communication and respect inside the couple (OR: 0,$3 ; 95 \% \mathrm{Cl}: 0,2-0,3$ ), and the fact that the woman has health insurance. Conclusions. Many individual factors, as well as factors of the couple relationship and others are associated with a higher probability of violence against the woman. Other agents, such as the communication and the respect in the couple, could have a protective character.
\end{abstract}

Key words: Domestic violence; Cultural diversity; Geographic locations; Health surveys; Violence against women; Peru (source: MeSH NLM).

\section{INTRODUCCIÓN}

La violencia de pareja contra la mujer es, con frecuencia, una de las causas prevenibles de morbilidad y mortalidad de la mujer en diversas culturas ${ }^{(1,2)}$. Existe evidencia de las graves consecuencias, directas e indirectas, que ocasiona la violencia de pareja en la salud de la mujer ${ }^{(2-5)}$. La prevalencia de violencia física o sexual contra la mujer por la pareja es alta aunque puede variar según los ámbitos geográficos. Estudios como los realizados por Hindin en diez países ${ }^{(6)}$ y por García-Moreno y su equipo en 2006, en quince sitios ubicados en diez países, dos de ellos

1 Facultad de Salud Pública, Universidad Peruana Cayetano Heredia. Lima, Perú.

a Médico, magíster en Salud Pública, doctor en Salud Pública; ${ }^{\text {b }}$ socióloga, magíster en Sociología, PhD en Antropología

* El estudio se originó a partir de la tesis de doctorado en Salud Pública sustentada por DBW en la Universidad Peruana Cayetano Heredia en 2011.

Recibido: 07-01-12 Aprobado: 22-02-12 
en Perú ${ }^{(7)}$, coinciden en la identificación de una elevada prevalencia de violencia física o sexual de pareja hacia la mujer, que varía entre 15 a $78 \%$ entre países e incluso entre diferentes sitios del mismo país ${ }^{(7)}$. En otros estudios realizados en el Perú por el INEI ${ }^{(8,9)}$ y el Instituto Nacional de Salud Mental ${ }^{(10)}$, también muestran esta variación entre los diferentes ámbitos geográficos o áreas de residencia.

La manifestación de la violencia de pareja es característica de cada cultura ${ }^{(1)}$. En el Perú hay una gran diversidad de culturas ${ }^{(11)}$ que son resultado de su adecuación a contextos geográficos específicos y están condicionadas por diferentes economías y ecologías. Estas diferencias han dado lugar a una variedad de sistemas culturales de reglas, normas, significados y expectativas compartidas, incluidos los papeles de género, la división sexual del trabajo, la división sexual del poder, las normas sociales de género, la representación pública respecto a la violencia y el honor, etc. ${ }^{(12)}$. A través de importantes estudios cualitativos realizados en el país se ha descrito diferencias en los tipos de relaciones de pareja y violencia en poblaciones según características culturales y sociales específicas ${ }^{(13-21)}$.

En el presente trabajo se consideraron dos teorías para construir el marco conceptual de aproximación al problema. La primera es la teoría ecológica ${ }^{(22,23)}$, que especifica que para entender la violencia se toma en cuenta la interacción de factores de diferentes niveles, que se relacionan con la dinámica de esta (víctimaperpetrador); estos niveles son: 1) individual (historia personal y factores biológicos), 2) de relaciones de pareja, 3) de relaciones con otros, y 4) sociales. La segunda, es la teoría sociológica de Bourdieu que considera las formas culturales aceptadas de dominación masculina ${ }^{(13,24)}$, como los papeles aceptados socialmente para hombres y mujeres, así como pautas para identificar los factores relacionados con la violencia física de la pareja hacia la mujer. Basados en estas teorías se realizó la categorización de variables y la definición de los ámbitos geográficos sobra la base de estudios cualitativos nacionales ${ }^{(13-21)}$.

Así, el objetivo del presente estudio es identificar la prevalencia de violencia física reciente (en el último año) de pareja y los factores asociados en el Perú, usando la información de la encuesta demográfica y de salud familiar 2004-2007 (ENDES continua).

\section{MATERIALES Y MÉTODOS}

Se realizó un análisis secundario de la base de datos de la encuesta demográfica y de salud familiar 2004-2007 (ENDES continua) conducida por el Instituto Nacional de Estadística e Informática (INEI). La descripción detallada de la encuesta y los procedimientos se encuentran publicados en la página web del INEI ${ }^{(25)}$. La muestra para este estudio fue una muestra probabilística, de áreas, estratificada, trietápica y autoponderada, sin reemplazo de mujeres en edad fértil (15 a 49 años de edad). Alcanzando una tasa de respuesta de $96,9 \%$.

La muestra total de la ENDES de los años 2004 al 2007 fue de 25489 mujeres. Como criterio de selección para este estudio se incluyeron mujeres que informaron tener una pareja en el momento de la realización de la encuesta y que respondieron al módulo de violencia. La muestra efectiva para el análisis fue de 12257 mujeres $(48,1 \%)$.

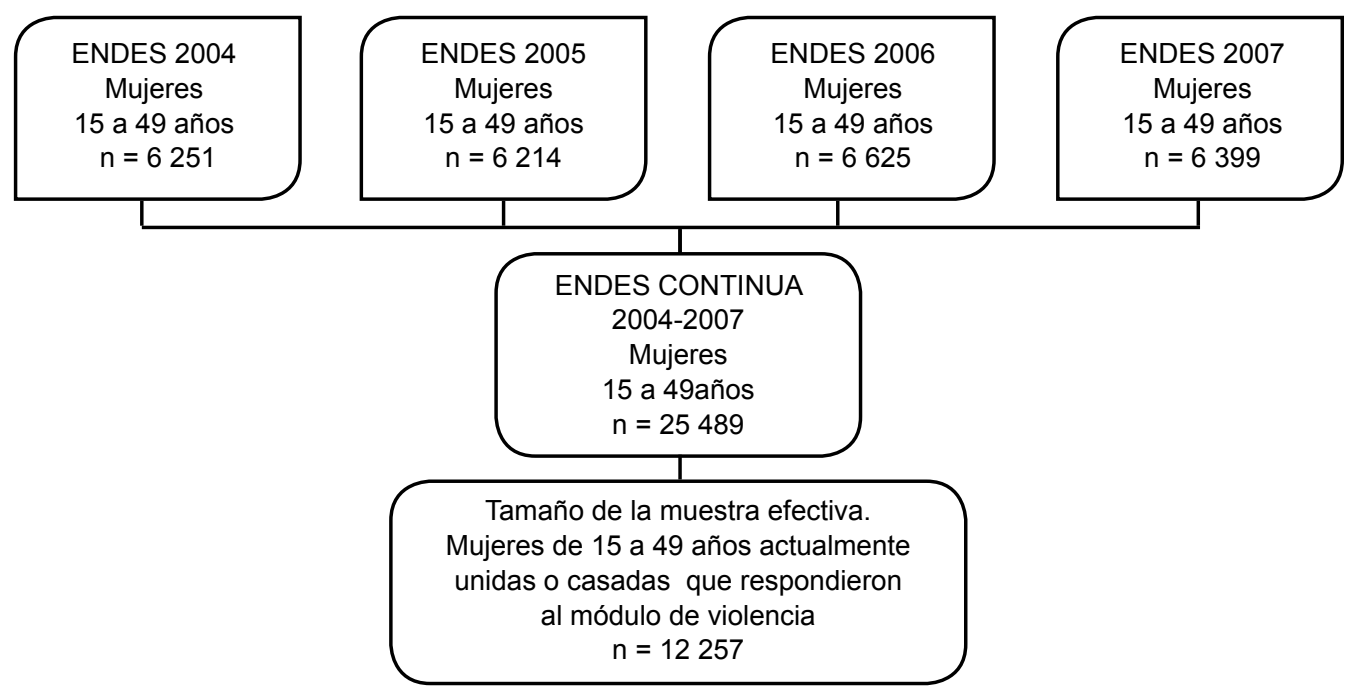

Figura 1. Flujograma de selección de la muestra efectiva para el estudio de la violencia física de la pareja contra la Mujer (ENDES Continua 2004-2007). 
La variable respuesta del estudio fue "violencia física reciente (en los últimos doce meses) por la pareja"; que se construyó a partir del informe proporcionado por la mujer de haber sufrido cualquier tipo de agresión física por su pareja. Se midió mediante una escala de nueve preguntas, que eran: su (último) esposo (marido) ¿la empujó, sacudió o le tiró algo?, ¿la abofeteó o le retorció el brazo?, ¿la golpeó con el puño o con algo que pudo hacerle daño?, ¿la ha pateado o arrastrado?, ¿trató de estrangularla o quemarla?, ¿la atacó/agredió con un cuchillo?, ¿la amenazó con un cuchillo, pistola u otro tipo de arma?, ¿ha utilizado la fuerza física para obligarla a tener relaciones sexuales, aunque usted no quería?, ¿la obligó a realizar actos sexuales que usted no aprueba? Si la respuesta era: "sí ", se preguntaba ¿con qué frecuencia en los últimos doce meses?, y si era "no", se pasaba a la siguiente pregunta.

Para el estudio, la violencia física reciente se categorizó como "sin violencia física" y "cualquier tipo de violencia física" (incluida la violencia física sexual), ya sea frecuente, o algunas veces en los últimos doce meses.

Además, se categorizaron variables y se determinó la consistencia interna de algunas escalas según el marco conceptual, los estudios cualitativos nacionales ${ }^{(13-21)}$ y a partir de la ENDES; se presentan las variables independientes, según niveles.

La variables del "nivel individual" fueron: antecedente de agresión física de su padre a su madre, edad a la primera unión, edad a la primera relación sexual, antecedente de relaciones previas de pareja, edad de la mujer, nivel de educación, lengua materna, número de hijos nacidos vivos.

Las del "nivel de relaciones de pareja" fueron: tipo de relación con pareja actual; tipo de comunicación con la pareja (para medir esta, se utilizó una escala basada en las respuestas acerca de si en la relación con su pareja reciente, él es cariñoso con ella, pasa su tiempo libre con ella, consulta su opinión en diferentes temas del hogar, respeta sus deseos, respeta sus derechos); consumo de alcohol por la pareja (se construyó a partir de las preguntas ¿su pareja toma alcohol? y ¿cuántas veces su pareja se emborracha?); toma de decisiones en la pareja (se consideraron preguntas sobre quién tiene la última palabra en las decisiones domésticas acerca del uso de métodos anticonceptivos, cómo se gasta el dinero, visitas a familiares, cuidado de salud de la mujer, compras grandes, compras y comidas de cada día. A partir de las respuestas se categorizó a las parejas en cuatro grupos de relaciones de poder: igualitaria, poder dividido, dominancia femenina o dominancia masculina); y diferencia en educación en la pareja (se creó restando el nivel máximo de educación alcanzado por la mujer con respecto al del hombre).

Las variables en el "nivel de relaciones con otros", fueron: control por la pareja de visitas a familiares y amigos (se utilizó una escala a partir de respuestas acerca de si la pareja se pone celoso o molesto si conversa con otro hombre, la acusa de ser infiel, limita las visitas/contactos con su familia o amigos, insiste en saber dónde va, desconfía de ella con el dinero); tolerancia social a la violencia (se uso una escala a partir del número de respuestas que justifican que la pareja golpee a la mujer; se le preguntó si en su opinión estaba de acuerdo en que la pareja golpee a su esposa en alguno o varios de los casos siguientes: si sale de la casa sin decirle nada a él, si descuida a los niños, si discute con él, si se niega a tener relaciones sexuales o si quema la comida). Mientras, en el "nivel social" se consideró al acceso a algún tipo de seguro en salud.

Se validó la consistencia interna (confiabilidad) de las escalas utilizadas para medir tipo de comunicación, toma de decisión en la pareja, control por la pareja y tolerancia social a la violencia, empleando el Alfa de Cronbach, siendo este valor de 0,$76 ; 0,67 ; 0,62$ y 0,69 respectivamente.

Se hizo un análisis descriptivo para establecer la distribución de las variables. Asimismo, se realizo regresión logística univariada con el fin de describir la asociación entre "violencia física de la pareja contra la mujer" y los diferentes niveles de análisis (como las variables correspondientes a nivel individual, de relaciones de pareja, de relaciones con otros, características socioeconómicas y ámbito geográfico). En el análisis multivariado se utilizó como criterio, el marco conceptual descrito previamente para este estudio. Se utilizó un modelo multivariado para modelar los factores significativos $(p<0,001)$ que operan en el mismo nivel conceptual o etapa del modelo. Las variables del nivel individual se introdujeron en primer lugar, seguido por los de nivel de relaciones de pareja, relaciones con otros y finalmente, lugar de residencia.

Todos los análisis se hicieron con métodos para muestras complejas, ajustando además por el número de hijos, año de la ENDES y edad de la mujer entrevistada. Los datos se analizaron utilizando el programa STATA 9. En este trabajo, el nivel de significancia fue de 0,05.

\section{RESULTADOS}

La proporción ponderada de violencia física reciente contra la mujer por su pareja en los últimos 12 meses, a nivel nacional, fue de 14,5\% (IC 95\% 13,6-15,3), 
Tabla 1. Características de la violencia física reciente contra la mujer por la pareja actual; datos seleccionados de la ENDES Continua 2004-2007

\begin{tabular}{|c|c|c|c|c|c|}
\hline \multirow{3}{*}{ Características de la muestra } & \multicolumn{4}{|c|}{$\begin{array}{c}\text { Violencia física por pareja actual } \\
\text { en los últimos doce meses }\end{array}$} & \multirow{3}{*}{ Valor $p$} \\
\hline & \multicolumn{2}{|c|}{ sí (N. $\left.{ }^{\circ}=1941\right)$} & \multicolumn{2}{|c|}{ NO $\left(N .{ }^{\circ}=10316\right)$} & \\
\hline & $\mathbf{N}^{*}$ & $(\%) * *$ & $\mathbf{N}^{*}$ & $(\%)^{* *}$ & \\
\hline \multicolumn{6}{|l|}{ NIVEL INDIVIDUAL } \\
\hline Grupo de edad (en años) & & & & & $<0,001$ \\
\hline $15-19$ & 93 & $(21,4)$ & 336 & $(78,6)$ & \\
\hline $20-24$ & 312 & $(20,5)$ & 1199 & $(79,5)$ & \\
\hline $25-29$ & 376 & $(15,3)$ & 1891 & $(84,7)$ & \\
\hline $30-34$ & 409 & $(14,0)$ & 2161 & $(86,0)$ & \\
\hline $35-39$ & 335 & $(14,0)$ & 1948 & $(86,0)$ & \\
\hline $40-44$ & 260 & $(12,6)$ & 1557 & $(87,5)$ & \\
\hline $45-49$ & 156 & $(10,1)$ & 1224 & $(89,9)$ & \\
\hline Lenguaje materno & & & & & 0,05 \\
\hline Español o castellano & 807 & $(13,5)$ & 4688 & $(86,5)$ & \\
\hline Quecha & 571 & $(14,3)$ & 2657 & $(83,7)$ & \\
\hline Aymara & 59 & $(11,6)$ & 397 & $(88,5)$ & \\
\hline Otros lenguajes indígenas & 20 & $(13,7)$ & 123 & $(86,3)$ & \\
\hline Lenguajes extranjeros & 2 & $(22,5)$ & 19 & $(77,5)$ & \\
\hline No respondió & $47 \overline{6}$ & $(29,9)$ & 2404 & $(70,1)$ & \\
\hline Antecedente de agresión física de su padre a su madre & & & & & $<0,001$ \\
\hline Sí & 1133 & $(18,8)$ & 4444 & $(81,2)$ & \\
\hline No & 699 & $(10,8)$ & 5234 & $(89,2)$ & \\
\hline Relaciones previas de pareja & & & & & $<0,001$ \\
\hline No & 1625 & $(13,8)$ & 9268 & $(86,3)$ & \\
\hline Sí & 316 & $(21,0)$ & 1048 & $(79,0)$ & \\
\hline \multicolumn{6}{|l|}{ NIVEL DE RELACIONES CON LA PAREJA } \\
\hline Relación con la pareja actual & & & & & $<0,001$ \\
\hline Casada & 642 & $(10,1)$ & 5086 & $(89,9)$ & \\
\hline Convive & 1299 & $(18,6)$ & 5230 & $(81,4)$ & \\
\hline Comunicación con la pareja & & & & & $<0,001$ \\
\hline No & 366 & $(43,4)$ & 471 & $(56,6)$ & \\
\hline Sí & 1575 & $(12,4)$ & 9845 & $(87,6)$ & \\
\hline Consumo de alcohol por la pareja & & & & & $<0,001$ \\
\hline No consume alcohol & 260 & $(8,5)$ & 2736 & $(91,5)$ & \\
\hline Toma alcohol pero no se embriaga & 95 & $(7,1)$ & 1008 & $(93,0)$ & \\
\hline Toma alcohol y se embriaga algunas veces & 1223 & $(15,3)$ & 6231 & $(84,7)$ & \\
\hline Toma alcohol y se embriaga seguido & 363 & $(50,4)$ & 337 & $(49,6)$ & \\
\hline Toma de decisiones en la pareja & & & & & $<0,001$ \\
\hline Decisión conjunta & 593 & $(11,1)$ & 4227 & $(88,9)$ & \\
\hline Predominantemente decide el hombre & 883 & $(17,5)$ & 3656 & $(82,5)$ & \\
\hline Predominantemente decide la mujer & 221 & $(15,2)$ & 1120 & $(84,8)$ & \\
\hline Toma dividida de decisiones & 244 & $(14,2)$ & 1313 & $(85,8)$ & \\
\hline Diferencia en nivel de educación con la pareja & & & & & $<0,001$ \\
\hline Ambos con mismo nivel & 821 & $(12,5)$ & 4920 & $(87,5)$ & \\
\hline Hombre con mayor nivel & 752 & $(15,4)$ & 3879 & $(84,7)$ & \\
\hline Mujer con mayor nivel & 364 & $(18,6)$ & 1503 & $(81,4)$ & \\
\hline \multicolumn{6}{|l|}{ NIVEL DE RELACIONES CON OTROS } \\
\hline $\begin{array}{l}\text { Control de las relaciones (familia amigos y otros) por la } \\
\text { pareja }\end{array}$ & & & & & $<0,001$ \\
\hline No & 199 & $(4,3)$ & 3804 & $(95,7)$ & \\
\hline Sí & 1742 & $(19,7)$ & 6512 & $(80,3)$ & \\
\hline $\begin{array}{l}\text { Tolerancia a la violencia física (la mujer considera justifica- } \\
\text { da la violencia física de un hombre a su mujer por alguna } \\
\text { circunstancia) }\end{array}$ & & & & & 0,20 \\
\hline No & 1331 & $(14,2)$ & 7359 & $(85,9)$ & \\
\hline Sí & 110 & $(16,5)$ & 450 & $(83,5)$ & \\
\hline Cuenta con algún tipo de seguro de salud & & & & & $<0,001$ \\
\hline No & 1547 & $(15,8)$ & 7430 & $(84,2)$ & \\
\hline Sí & 392 & $(11,0)$ & 2872 & $(89,0)$ & \\
\hline Ámbito geográfico & & & & & $<0,001$ \\
\hline Lima Metropolitana y Callao & 112 & $(12,0)$ & 820 & $(88,0)$ & \\
\hline Resto de costa urbano & 327 & $(14,6)$ & 2013 & $(85,4)$ & \\
\hline Sierra urbana & 334 & $(18,9)$ & 1385 & $(81,1)$ & \\
\hline Selva urbana & 274 & $(17,9)$ & 1207 & $(82,1)$ & \\
\hline Resto de costa rural & 63 & $(9,7)$ & 558 & $(90,3)$ & \\
\hline Sierra rural & 545 & $(14,4)$ & 2965 & $(85,7)$ & \\
\hline Selva rural & 286 & $(16,3)$ & 1368 & $(83,7)$ & \\
\hline
\end{tabular}

* Sin ponderar; ** Ponderado 
presentando variación en la proporción de esta en los diferentes ámbitos geográficos.

La proporción de mujeres víctimas de violencia física reciente de pareja fue para Lima Metropolitana 12,0\% (IC 95\% 9,7-14,4), resto de costa urbana 14,6\% (IC 95\% 12,8-16,4), en sierra urbana 18,9\% (IC 95\% 16,7-21,0), selva urbana $17,9 \%$ (IC 95\% 15,2- 20,5), en costa rural 9.6\% (IC 95\% 7,7-11,7), en sierra rural 14,4\% (IC 95\% $12,6-16,1)$ y el selva rural 16,3\% (IC 95\% 13,8-18,9). La Tabla 1 también muestra la distribución de las variables por presencia o no de violencia física reciente por parte de la pareja.
Las mujeres que informaron violencia física reciente comparadas con las que no, son más jóvenes (mediana de edad 32,1 frente a 33,6 años), con edad más temprana de inicio de relaciones sexuales (mediana de edad 19,3 frente a 20 años) y de primera unión de pareja (mediana de la edad 19,7 frente a 21,4 años).

La Tabla 2 muestra la mayor parte de los factores asociados con la violencia física reciente por la pareja, tanto en el modelo no ajustado (crudo) y el modelo multivariado (ajustado). Entre estos factores, los que mantuvieron asociación estadística en el modelo multivariado con violencia física fueron: la presencia de

Tabla 2. Factores asociados a la violencia física reciente de la pareja* hacia la mujer: factores individuales, de relaciones de pareja, de relaciones con otros, sociales y ámbitos geográficos; 2004-2007.

\begin{tabular}{|c|c|c|c|c|c|c|}
\hline \multirow{2}{*}{ Variables asociadas } & \multicolumn{3}{|c|}{ Asociación cruda } & \multicolumn{3}{|c|}{ Asociación ajustada } \\
\hline & OR & (IC 95\%) & $\mathrm{p}$ & OR & $(\mathrm{IC} 95 \%)$ & $\mathrm{p}$ \\
\hline \multicolumn{7}{|c|}{ Antecedente de violencia física del padre contra la madre de la mujer } \\
\hline No & Ref. & & & Ref. & & \\
\hline Sí & 1,9 & $(1,7-2,2)$ & $<0,001$ & 1,7 & $(1,4-1,9)$ & $<0,001$ \\
\hline \multicolumn{7}{|l|}{ Toma de decisiones en la pareja } \\
\hline Decisión conjunta & Ref. & & & Ref. & & \\
\hline Decide predominantemente mujer & 1,7 & $(1,5-2,0)$ & $<0,001$ & 1,2 & $(1,05-1,5)$ & 0,011 \\
\hline Decide predominantemente hombre & 1,4 & $(1,2-1,8)$ & 0,001 & 1,2 & $(0,97-1,6)$ & 0,085 \\
\hline Decisión dividida & 1,3 & $(1,1-1,6)$ & 0,011 & 1,1 & $(0,90-1,5)$ & 0,263 \\
\hline \multicolumn{7}{|l|}{ Consumo de alcohol por la pareja } \\
\hline No toma bebidas alcohólicas & Ref. & & & Ref. & & \\
\hline Pareja toma alcohol pero no se embriaga & 0,8 & $(0,6-1,1)$ & 0,212 & 0,9 & $(0,6-1,3)$ & 0,56 \\
\hline Pareja toma alcohol y se embriaga a veces & 1,9 & $(1,6-2,3)$ & $<0,001$ & 1,8 & $(1,5-2,2)$ & $<0,001$ \\
\hline Pareja toma alcohol y se embriaga seguido & 10,9 & $(8,4-14,2)$ & $<0,001$ & 7,2 & $(5,4-9,6)$ & $<0,001$ \\
\hline \multicolumn{7}{|c|}{ Control o limitación por la pareja de visitas a familia, amigos y otros } \\
\hline No & Ref. & & & Ref. & & \\
\hline Sí & 5,5 & $(4,5-6,6)$ & $<0,001$ & 4,1 & $(3,3-5,0)$ & $<0,001$ \\
\hline \multicolumn{7}{|c|}{ Comunicación y respeto. Pareja muestra interés en su opinión o estar con usted o cariño o respeto } \\
\hline No & Ref. & & & Ref. & & \\
\hline Sí & 0,2 & $(0,15-0,2)$ & $<0,001$ & 0,3 & $(0,2-0,3)$ & $<0,001$ \\
\hline \multicolumn{7}{|l|}{ Seguro de salud de la mujer } \\
\hline No & Ref. & & & Ref. & & \\
\hline Sí & 0,7 & $(0,6-0,8)$ & $<0,001$ & 0,8 & $(0,7-1,0)$ & 0,036 \\
\hline \multicolumn{7}{|l|}{ Tipo de relación con la pareja } \\
\hline Casada & Ref. & & & Ref. & & \\
\hline Conviviente & 2,0 & $(1,8-2,3)$ & $<0,001$ & 1,4 & $(1,2-1,6)$ & $<0,001$ \\
\hline \multicolumn{7}{|l|}{ Relaciones previas de pareja de la mujer } \\
\hline No & Ref. & & & Ref. & & \\
\hline Sí & 1,7 & $(1,4-2,0)$ & $<0,001$ & 1,4 & $(1,1-1,7)$ & 0,008 \\
\hline \multicolumn{7}{|c|}{ Diferencia de nivel de educación de la mujer con el de la pareja } \\
\hline Igual nivel & Ref. & & & Ref. & & \\
\hline Menor nivel de educación que su pareja & 1,3 & $(1,1-1,5)$ & 0,001 & 1,1 & $(0,9-1,3)$ & 0,286 \\
\hline Mayor nivel de educación que su pareja & 1,6 & $(1,3-1,9)$ & $<0,001$ & 1,4 & $(1,1-1,7)$ & 0,003 \\
\hline Edad de la mujer en años & 0,97 & $(0,97-0,98)$ & $<0,001$ & 0,97 & $(0,96-0,98)$ & $<0,001$ \\
\hline Número de hijos de la mujer & 1,00 & $(0,97-1,03)$ & 0,968 & 1,0 & $(0,98-1,1)$ & 0,349 \\
\hline \multicolumn{7}{|l|}{ Lugar de residencia de la mujer } \\
\hline Lima Metropolitana y Callao & Ref. & & & Ref. & & \\
\hline Resto de costa urbana & 1,2 & $(1,0-1,6)$ & 0,096 & 1,3 & $(1,01-1,8)$ & 0,044 \\
\hline Sierra urbana & 1,7 & $(1,3-2,2)$ & $<0,001$ & 1,5 & $(1,1-1,9)$ & 0,009 \\
\hline Selva urbana & 1,6 & $(1,2-2,1)$ & 0,001 & 1,3 & $(0,9-1,8)$ & 0,103 \\
\hline Resto de costa rural & 0,8 & $(0,6-1,1)$ & 0,128 & 0,8 & $(0,5-1,2)$ & 0,353 \\
\hline Sierra rural & 1,2 & $(0,9-1,6)$ & 0,126 & 1,1 & $(0,9-1,5)$ & 0,345 \\
\hline Selva rural & 1,4 & $(1,1-1,9)$ & 0,015 & 1,0 & $(0,7-1,4)$ & 0,842 \\
\hline \multicolumn{7}{|l|}{ Año de entrevista } \\
\hline 2004 & Ref. & & & Ref. & & \\
\hline 2005 & 1,0 & $(0,8-1,2)$ & 0,764 & 1,0 & $(0,8-1,2)$ & 0,706 \\
\hline 2006 & 0,9 & $(0,7-1,1)$ & 0,359 & 1,0 & $(0,8-1,2)$ & 0,651 \\
\hline 2007 & 1,00 & $(0,8-1,2)$ & 0,991 & 1,0 & $(0,8-1,2)$ & 0,811 \\
\hline
\end{tabular}

*En los últimos doce meses 
antecedentes de que el padre haya golpeado a la madre de la participante $(p<0,001)$, el que hayan tenido una o más relaciones de pareja previa a la actual $(p=0,008)$, el que su estado civil actual sea "conviviente" $(p<0,001)$ y que haya uso de alcohol hasta la embriaguez por parte de la pareja.

Específicamente, las mujeres con parejas que toman alcohol hasta la embriaguez frecuentemente, tienen siete veces más riesgo de sufrir violencia física por su pareja que las que no lo tienen (OR: 7,2; IC 95\%: 5,4$9,6 ; p<0,001)$. Además, las mujeres con parejas que las controlan haciendo que estas limiten su interacción con la familia o amigos tienen cuatro veces más posibilidad de sufrir violencia física por su pareja que las que no son controladas por sus parejas (OR: 4,1; IC 95\%: 3,3-5,0; $p<0,001)$. Por otra parte, los factores que se asociaron con un menor riesgo de ser víctima de violencia física de pareja incluyeron la edad de la mujer en años (mayor edad que el varón) ( $p<0,001)$, la comunicación y respeto en la relación de convivencia con la pareja (OR: 0,3; IC 95\%: 0,2-0,3; $p<0,001$ ) y que la mujer tenga algún tipo de seguro de salud $(p=0,04)$.

Adicionalmente, el modelo multivariado encontró como factores asociados a mayor riesgo para violencia por la pareja, el que la mujer tenga mayor educación que el hombre, el que sea predominantemente la mujer la que toma las decisiones en pareja y residir en un lugar dentro del resto de la costa urbana o en la sierra urbana peruanas. Es importante notar que, en general, las asociaciones entre el modelo crudo y ajustado coinciden.

\section{DISCUSIÓN}

Los principales hallazgos de este estudio incluyen la evidencia de una elevada proporción de violencia física reciente de pareja hacia la mujer, la cual puede variar a través de los diferentes ámbitos geográficos en el Perú (por ejemplo, de $18,9 \%$ en sierra urbana y $9,7 \%$ en resto de costa rural). Conforme a las teorías de Heise y Bronfenbrenner ${ }^{(22,23)}$ y de Bourdieu ${ }^{(24)}$, se ha identificado diferentes factores asociados con violencia física de pareja contra la mujer, que se relacionan con las normas tradicionales de género o "machistas", tales como el control por el hombre de visitas a familiares y amigos de la mujer; que el varón se sienta amenazado porque ella tuvo una unión previa de pareja; que la mujer tenga más nivel de educación que él o que ella tome la mayor parte de decisiones en el hogar. Este hallazgo sugiere que es importante involucrar a varones y mujeres en la elaboración de estrategias que incluyan el análisis y cuestionamiento de este tipo de normas e identidades de género $\mathrm{y}$, consecuentemente, propiciar la construcción de acuerdos de comportamientos aceptables entre hombres y mujeres, que prevengan situaciones de violencia.

Por otra parte, el consumo excesivo de alcohol, que en nuestro país ${ }^{(2,26)}$ al igual que en otros países y contextos ${ }^{(6,27)}$, es una característica relacionada con roles sociales masculinos, se encuentra asociado de manera consistente a la violencia física contra la mujer ${ }^{(27)}$. Ello sugiere la importancia de implementar políticas públicas y medidas multisectoriales que incluyan todo nivel de servicios de salud, educación y comunitarios además del tamizaje, identificación y derivación a tratamiento de aquellos individuos que presentan un consumo problemático de alcohol. Es necesaria, además, la creación de normas comunitarias para prevenir que el uso de alcohol sea utilizado como excusa moral o legal para justificar la violencia de pareja ${ }^{(28)}$. Este es un factor clave en la prevención de la violencia de pareja puesto que desarrollar intervenciones que incluyan cambios de normas culturales sobre el uso excesivo de alcohol tiene repercusión directa en la prevención primaria de la violencia de pareja ${ }^{(7)}$.

El antecedente de violencia del padre sobre la madre ha sido documentado como un factor asociado a riesgo en múltiples lugares y países, incluido el Perú $(2,26,27)$. Según la teoría de aprendizaje social de Bandura ${ }^{(29)}$, esta asociación se explica porque aquellas mujeres que de niñas fueron testigos de violencia entre sus padres aprenden que la violencia en las relaciones de pareja es algo habitual y, por lo tanto, es más probable que en etapas posteriores de su vida, se vean involucradas en tales situaciones. Es por ello que se hace necesario investigar y validar estrategias de identificación de este fenómeno en etapas tempranas de la vida, para prevenir y atenuar situaciones de violencia en el hogar. Se resalta la necesidad de una respuesta integrada a este problema que se presenta de manera cíclica en diferentes generaciones y que puede tener importantes implicaciones en la reducción del riesgo de violencia en etapas posteriores de la vida. Es por ello necesario el trabajo entre sectores y diferentes programas y actores comunitarios.

Varios estudios internacionales han documentado una mayor tasa de violencia física de la pareja contra la mujer si son convivientes en lugar de estar casadas ${ }^{(30,31)}$. En el Perú, si la mujer es conviviente tiene un incremento de riesgo de violencia física contra ella por la pareja a lo largo de su vida ${ }^{(27)}$ y en los últimos doce meses. En Latinoamérica, la coexistencia de matrimonio y convivencia como patrón de formación familiar es un rasgo distintivo, siendo esta última más inestable y con 
posibilidad de disolución más frecuente. En el Perú, la convivencia no se encuentra confinada a un periodo transitorio durante la juventud, sino que es mucho más permanente y se presenta en todas las etapas de la vida, con tendencia a generalizarse debido a tradiciones culturales y a consideraciones de índole económica, particularmente en generaciones más jóvenes ${ }^{(28)}$. Por eso es necesario identificar las características del patrón de unión de parejas en el país, dentro de los diferentes ámbitos geográficos para proponer y validar estrategias y normas que eviten el aislamiento de la mujer y las condiciones que favorecen la violencia contra ella, así como mecanismos preventivos de identificación y resolución de los conflictos generados por la violencia de pareja.

En el Perú se ha identificado previamente un mayor riesgo de violencia física de la pareja a lo largo de la vida de la mujer asociado con residir en lugares diferentes a la costa (27). El considerar ámbitos geográficos de residencia en este estudio permitió identificar una asociación a mayor riesgo de violencia física de la pareja contra la mujer de ámbitos geográficos urbanos de resto de costa y sierra peruanas. Este hallazgo coincide con información de estudios nacionales cuantitativos y cualitativos sobre una mayor frecuencia de violencia física en la sierra ${ }^{(8,10,15-17,19)}$. Una posible explicación a ello viene de estudios cualitativos que han mostrado que los procesos de transformación personal y colectiva de identidades y los cambios en las normas de género, tales como el acceso a mayores niveles de educación en la mujer y el formar parte de la fuerza laboral, son percibidos por los varones como una amenaza hacia su masculinidad y su papel de proveedores únicos; mientras que en las mujeres, el problema de la transición del eje de su realización personal (sólo como madre y ama de casa) hacia su desarrollo profesional (trabajo remunerado) implica una sobrecarga que trae consigo el doble papel que debe cumplir muchas veces de manera simultánea ${ }^{(16,19)}$. Estos cambios acarrean el incumplimiento de las normas de género y de las expectativas culturales consideradas necesarias para ser una buena mujer o un hombre exitoso ${ }^{(17,19,20)}$. Éstas son las situaciones que Jewkes resalta como desencadenadoras de la violencia de pareja ${ }^{(32)}$, información que permite generar nuevas hipótesis acerca de la dinámica de la violencia de pareja dentro de estos ámbitos geográficos, las que deben ser investigadas a través estudios cuali-cuantitativos ad hoc.

El presente trabajo identificó, en el Perú, como factores asociados a protección de violencia física reciente de la pareja contra la mujer, la comunicación en la pareja y el que la mujer cuente con algún tipo de seguro de salud. Por lo tanto, la comunicación debe fomentarse a través de estrategias especialmente diseñadas para tal fin. Asimismo, el que la mujer tenga un seguro de salud puede estar relacionado con mayor independencia y más recursos comunitarios a los que puede recurrir. Esta información permite generar hipótesis para el desarrollo de estudios mixtos cuali-cuantitativos en los diferentes ámbitos geográficos del país y la validación de estrategias que incluyan la posibilidad de dar a las mujeres seguro de salud para la prevención de la violencia.

Es importante señalar las principales limitaciones de este estudio que merecen atención. Una de ellas es que la fuente de análisis de información (la ENDES continua 2004-2007), si bien provee de una muestra representativa de mujeres unidas en el Perú, no tuvo como objetivo principal la violencia de pareja, a pesar de utilizar la metodología del estudio multicéntrico de la OMS sobre la violencia de pareja y la salud de las mujeres ${ }^{(7)}$. Por esta razón, por su complejidad, la manera de medirla no incluye todas las dimensiones de esta (física, psicológica, etc.), no incluye a la pareja, ni tampoco un enfoque integral que tome en cuenta todos los factores que la expliquen, según la teoría ecológica. Adicionalmente, no se contemplan los factores contextuales ni las diferencias interpretativas que pueden interferir en las respuestas de las mujeres ${ }^{(1)}$. El diseño de este estudio (transversal) no permite establecer causalidad.

Para poder establecer causalidad en la violencia de pareja, dada su complejidad, se requiere que se utilicen en su investigación metodologías mixtas cualitativascuantitativas y estudios experimentales, donde se incluya además a ambos miembros de la pareja. Para tener una mejor comprensión, debe emplearse como modelo conceptual de diseño y análisis las teorías ecológicas y sociológicas que tomen en consideración dicha complejidad, así como las características culturales y normas e identidad de género en los diferentes ámbitos geográficos.

Asimismo, es importante incluir en futuros estudios a parejas adolescentes y el tema de violencia en el noviazgo o enamoramiento, puesto que la dinámica de pareja se inicia en etapas más tempranas de la vida, aun antes de la convivencia o matrimonio. Por ello, las medidas preventivas resultantes del conocimiento de dichas etapas tempranas pueden resultar en beneficio a mediano y largo plazo.

En conclusión, este estudio proporciona evidencia consistente de que existen diversas características individuales y de relaciones de pareja, con otros, sociales y factores geográficos que están asociados con una mayor probabilidad de violencia de pareja 
contra la mujer. Otros factores, como la comunicación y respeto en la pareja, podrían tener carácter protector. Esta información puede ayudar a informar programas o políticas públicas que tengan la finalidad de reducir los efectos negativos en la salud producidos por la violencia física de pareja contra la mujer.

\section{AGRADECIMIENTOS}

Al Ing. Yomtov Levy Spack por su esfuerzo y compromiso con este proyecto, al Mg. Miguel Ramos, Dr. Cesar Cárcamo, Dr. Carlos Cáceres, a la Lic. María Eloísa Núñez Robles y al Dr. Fabián Fiestas por sus sugerencias y aportes para la publicación de este articulo, así como a Martha y Graciela Álvarez por su ayuda en la revisión de estilo.

\section{Contribuciones de autoría}

DBW participó en la concepción y diseño del trabajo, en la recolección y obtención de resultados, en el análisis e interpretación de datos, en la redacción, en la revisión crítica y aprobó la versión final del manuscrito. ERS participó en el análisis e interpretación de datos, en la redacción, en la revisión crítica y en la aprobación final del manuscrito.

\section{Fuentes de financiamiento}

Autofinanciado.

\section{Conflictos de interés}

Las autoras no tienen ningún conflicto de interés en la publicación de este artículo.

\section{REFERENCIAS BIBLIOGRÁFICAS}

1. Fischbach RL, Herbert B. Domestic violence and mental health: correlates and conundrums within and across cultures. Soc Sci Med. 1997;45(8):1161-76.

2. Ellsberg $\mathrm{M}$, Jansen $\mathrm{H}$, Heise $\mathrm{L}$, Watts $\mathrm{CH}$, Garcia-Moreno C. Intimate partner violence and women's physical and mental health in the WHO multi-country study on women's health and domestic violence: an observational study. Lancet. 2008;371(9619):1165-72.

3. Devries KM, Kishor S, Johnson H, Stockl H, Bacchus LJ, Garcia-Moreno C, et al. Intimate partner violence during pregnancy: analysis of prevalence data from 19 countries. Reprod Health Matters. 2010;18(36):158-70.

4. Cripe SM, Sanchez SE, Gelaye B, Sanchez E, Williams MA. Association Between Intimate Partner Violence, Migraine and Probable Migraine. Headache. 2011;51(2):208-19.

5. Cripe SM, Sanchez SE, Perales MT, Lam N, Garcia P, Williams MA. Association of intimate partner physical and sexual violence with unintended pregnancy among pregnant women in Peru. Int $\mathrm{J}$ Gynaecol Obstet. 2008;100(2):104-8.

6. Hindin J, Kishor S, Ansara D. Intimate partner violence among couples in 10 DHS countries. RHM. 2008;17(33):1-75
7. Garcia-Moreno C, Jansen H, Ellsberg M, Heise L, Watts $\mathrm{CH}$. Prevalence of intimate partner violence: findings from the WHO multi-country study on women's health and domestic violence. Lancet. 2006;368(9543):1260-9.

8. Instituto Nacional de Estadística e Informática (INEI). Capitulo 12: Violencia contra la mujer, niñas y niños en Perú [Internet]. En: Encuesta Demográfica y de Salud Familiar 2010. Lima: INEI; 2011 [citado el 6 de febrero 2012]. Disponible en: http://proyectos.inei.gob.pe/endes/ endes2010/resultados/index.html

9. Instituto Nacional de Estadística e Informática (INEI). Modelos multivariados para la violencia conyugal,sus consecuencias y la solicitud de ayuda [Internet]. Lima: INEI; 2009 [citado el 6 de febrero 2012]. Disponible en: http://www1.inei.gob.pe/ biblioineipub/bancopub/Est/Lib0899/Libro.pdf.

10. Instituto Nacional de Salud Mental Honorio Delgado Hideyo Noguchi. Estudio Epidemiológico de Salud Mental en la Sierra Rural 2008. Anales de Salud Mental. 2009;25(1-2)

11. Comas-Diaz L, Lykes MB, Alarcon RD. Ethnic conflict and the psychology of liberation in Guatemala, Peru, and Puerto Rico. Am Psychol. 1998;53(7):778-92.

12. Cohen D. Cultural variation: Considerations and implications. Psychol Bull. 2001;127(4):451-71.

13. Reategui $R$, ed. Después de Michel Foucault: El poder, el saber, el cuerpo. Lima, Perú: SUR Casa de Estudios del Socialismo; 2006.

14. Ramos M. Masculinidades y violencia conyugal: Experiencias de vida de hombres de sectores populares de Lima y Cusco. Lima: FASPA- UPCH; 2006.

15. Fuller N. Contrastes regionales en las identidades de género en el Perú urbano. El caso de las mujeres de la baja Amazonía. Anthropologica. 2004;22(22):119-36.

16. Ruiz-Bravo P. Andinas y criollas: identidades femeninas en el medio rural peruano. En: Jerarquias en jaque: Estudios de género en el área andina. Fuller N, Editor. Lima: Red para el Desarrollo de las Ciencias Sociales en el Perúl CLACSO; 2004.p.283-319.

17. Meentzen A. Relaciones de género, poder e identidad femenina en cambio: el orden social de los aymaras rurales peruanos desde la perspectiva femenina. Cusco: Centro Bartolomé de Las Casas; 2007.

18. Valdeavellano EN, Riquelme E, editores. Foro Internacional Salud reproductiva en la Amazonia: Perspectivas desde la Cultura, el Género y la Comunicación. 1 ed. Lima: Minga; 2004.

19. Fuller N, editor. Jerarquías en jaque: estudios de género en el área andina. Lima: Red para el Desarrollo de las Ciencias Sociales en el Perú/ CLACSO; 2004.

20. Fuller N. Masculinidades cambios y permanencias. Lima Fondo Editorial Pontificia Universidad Católica del Perú; 2002.

21. Cáceres C, Salazar X, Rosasco AM, Fernández P. Ser hombre en el Perú de hoy: Una mirada a la salud sexual desde la infidelidad, la violencia y la homofobia. Lima, REDES Jóvenes; 2002.

22. Heise LL. Violence against women: an integrated, ecological framework. Violence Against Women. 1998;4(3):262-90.

23. Bronfenbrenner U. Ecology of the family as a context for human - development: research perspectives. Developmental Psychology. 1986;22(6):723-42. 
24. Bourdieu P. La dominación masculina. Barcelona: Editorial Anagrama; 2000.

25. Encuesta Demografica y de Salud Familiar [Internet]. Lima, Peru: Instituto Nacional de Estadistica e Informatica; 20002011 [citado 18 Feb 2012]. Disponible en: http://desa.inei. gob.pe/endes/documentos.asp

26. Flake DF. Individual, family, and community risk markers for domestic violence in Peru. Violence Against Women. 2005;11(3):353-73.

27. Abramsky T, Watts $\mathrm{CH}$, Garcia-Moreno C, Devries K, Kiss $\mathrm{L}$, Ellsberg $\mathrm{M}$, et al. What factors are associated with recent intimate partner violence? findings from the WHO multicountry study on women's health and domestic violence. BMC Public Health. 2011;11(109):1-17.

28. Graham K, Bernards S, Wilsnack SC, Gmel G. Alcohol may not cause partner violence but it seems to make it worse: a cross national comparison of the relationship between alcohol and severity of partner violence. J Interpers Violence. 2011;26(8):1503-23.
29. Bandura A, Barab PG. Conditions governing nonreinforced imitation. Developmental Psychology. 1971;5(2):244-55.

30. Brownridge DA, Halli SS. "Living in sin" and sinful living: Toward filling a gap in the explanation of violence against women. Aggression and Violent Behavior. 2000;5(6):56583.

31. Vandello JA, Cohen D. Male honor and female fidelity: Implicit cultural scripts that perpetuate domestic violence. $J$ Pers Soc Psychol. 2003;84(5):997-1010.

32. Jewkes R. Intimate partner violence: causes and prevention. Lancet. 2002;359(9315):1423-9.

Correspondencia: Dora Blitchtein

Dirección: Los Nogales 765 Dpto. 402, Lima 27, Perú.

Teléfono: (511) 4229826, 999090917

Correo electrónico: dblit2007@gmail.com

\section{Consulte las ediciones anteriores de la} Revista Peruana de Medicina Experimental y Salud Pública en www.scielo.org.pe

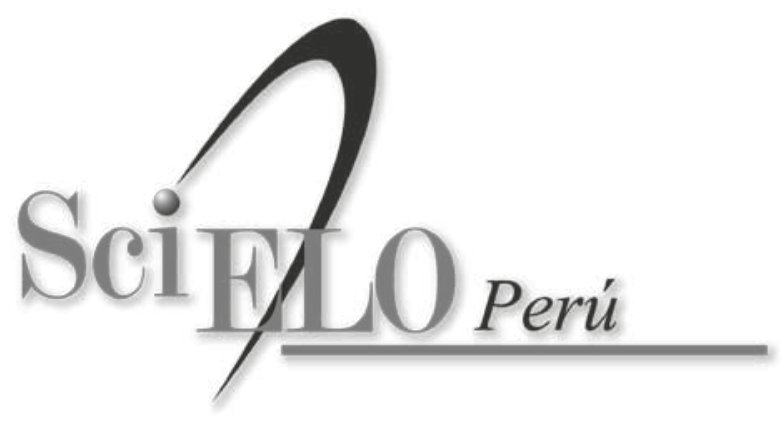

